\title{
ECOLOGICAL MONITORING AND COMPLIANCE PROGRAM FISCAL YEAR 2002 REPORT
}
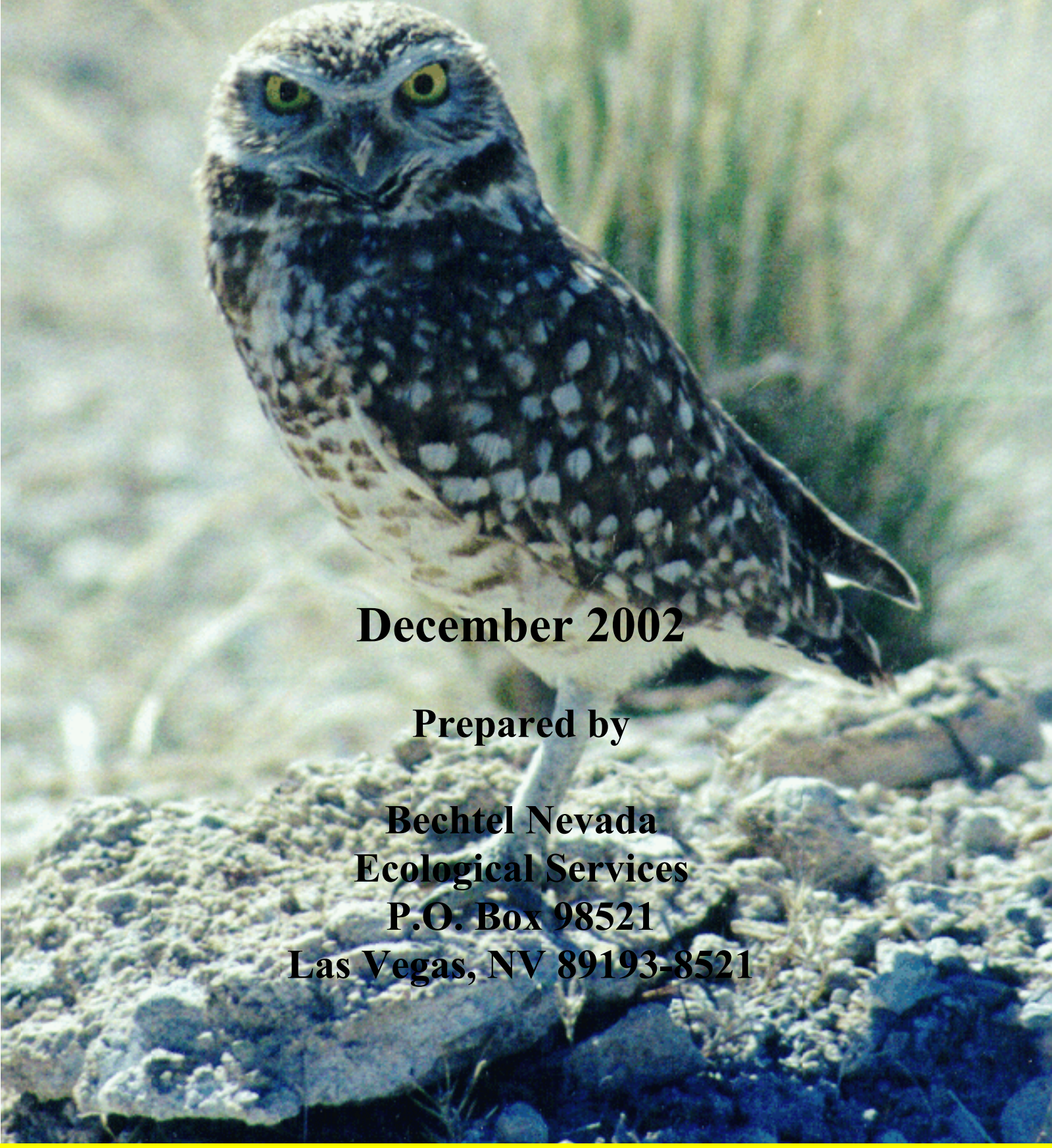
Reference herein to any specific commercial product, process, or service by trade name, trademark, manufacturer, or otherwise, does not necessarily constitute or imply its endorsement, recommendation, or favoring by the U.S. Government or any agency thereof or its contractors or subcontractors.

\section{AVAILABILITY STATEMENT}

Available for sale to the public from:

U.S. Department of Commerce

National Technical Information Service

5285 Port Royal Road

Springfield, VA 22161-0002

Telephone: 800-553-6847

Fax: 703-605-6900

E-mail: orders@ntis.fedworld.gov

Online ordering: http://www.ntis.gov/ordering.htm

Available electronically at http://www.doe.gov/bridge

Available for a processing fee to the U.S. Department of Energy and its contractors, in paper, from:

U.S. Department of Energy

Office of Scientific and Technical Information

P.O. Box 62

Oak Ridge, TN 37831-0062

Telephone: 865-576-8401

Fax: 865-576-5728

E-Mail: reports@adonis.osti.gov 


\title{
ECOLOGICAL MONITORING AND COMPLIANCE PROGRAM FISCAL YEAR 2002 REPORT
}

\author{
December 2002 \\ WORK PERFORMED UNDER \\ CONTRACT NO. DE-AC08-96NV11718 \\ Prepared for the \\ U.S. Department of Energy \\ National Nuclear Security Administration \\ Nevada Operations Office \\ Environment, Safety, and Health Division \\ P.O. Box 98518 \\ Las Vegas, NV 89193-8518
}

Prepared by

\section{Bechtel Nevada}

Ecological Services

P.O. Box 98521

Las Vegas, NV 89193-8521 
THIS PAGE IS INTENTIONALLY LEFT BLANK 


\section{CONTENTS}

ACRONYMS AND ABBREVIATIONS $\ldots \ldots \ldots \ldots \ldots \ldots \ldots \ldots \ldots \ldots \ldots$ vii

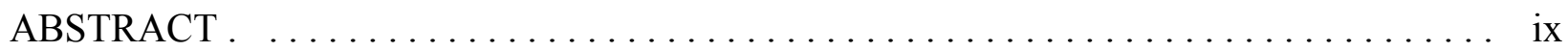

1.0 INTRODUCTION $\ldots \ldots \ldots \ldots \ldots \ldots \ldots \ldots \ldots \ldots \ldots \ldots \ldots \ldots \ldots \ldots \ldots \ldots$

2.0 BIOLOGICAL SURVEYS $\ldots \ldots \ldots \ldots \ldots \ldots \ldots \ldots \ldots \ldots \ldots \ldots \ldots \ldots \ldots \ldots$

2.1 Sites Surveyed and Sensitive Species Observed $\ldots \ldots \ldots \ldots \ldots \ldots \ldots \ldots$

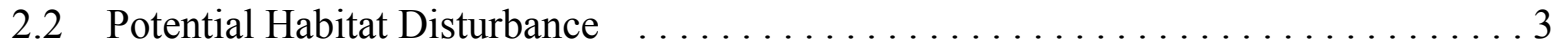

3.0 DESERT TORTOISE COMPLIANCE $\ldots \ldots \ldots \ldots \ldots \ldots \ldots \ldots \ldots \ldots \ldots \ldots \ldots \ldots$

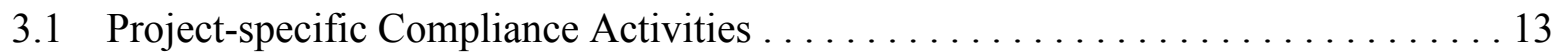

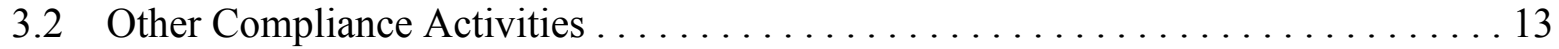

4.0 ECOSYSTEM MAPPING/DATA MANAGEMENT $\ldots \ldots \ldots \ldots \ldots \ldots \ldots \ldots \ldots \ldots \ldots$

4.1 Compilation of Historical Wildlife Data $\ldots \ldots \ldots \ldots \ldots \ldots \ldots \ldots \ldots$

4.2 NTS Vegetation Classification Metadata . . . . . . . . . . . . . . . . . 17

4.3 Coordination With Ecosystem Management Agencies/Scientists . . . . . . . . . 18

5.0 SENSITIVE SPECIES AND HABITAT MONITORING $\ldots \ldots \ldots \ldots \ldots \ldots \ldots \ldots \ldots$

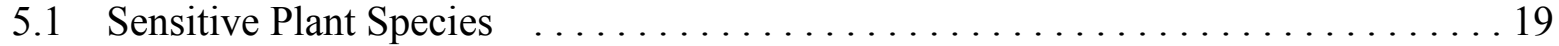

5.1.1 Revised List of Sensitive Plant Species for the NTS . . . . . . . . . . 19

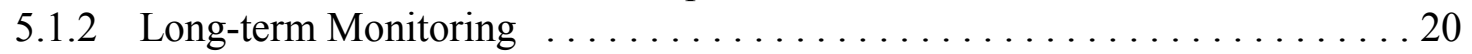

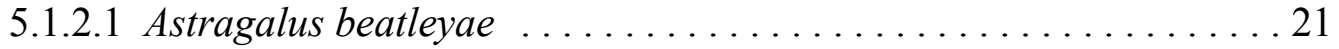

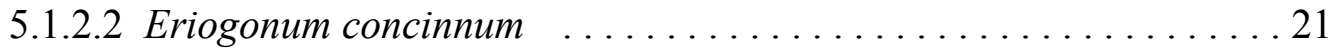

5.1.3 Coordination With Natural Resource Agency Botanists . . . . . . . . . 25

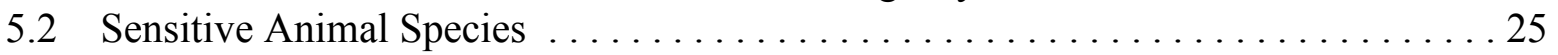

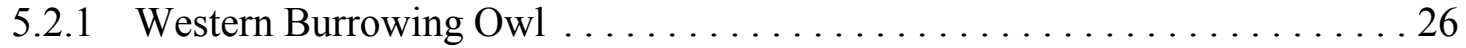

5.2.1.1 Burrow Distribution ....................... 26

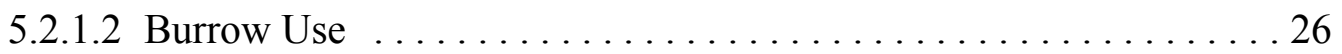

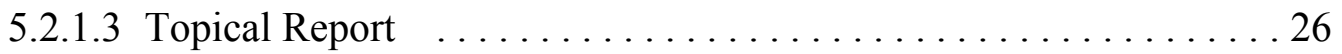

5.2.1.4 Coordination With Other Wildlife Agencies/Biologists . . . . . . . 28

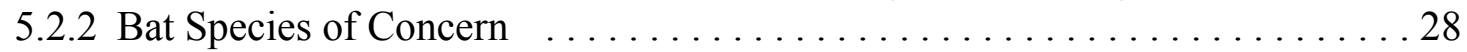

5.2.2.1 Building Roost Site Surveys . . . . . . . . . . . . . . 28

5.2.2.2 Coordination With Other Wildlife Agencies/Biologists . . . . . . . 28

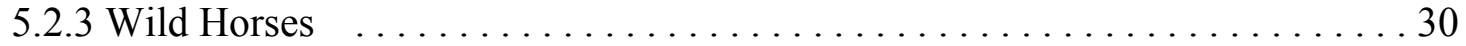

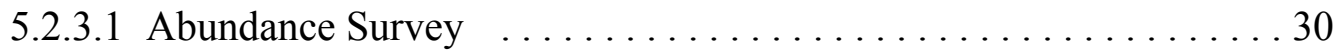

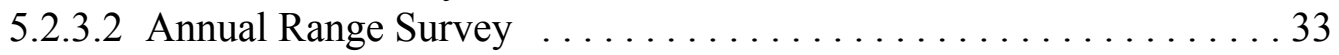

5.2 .3 .3 Use of NTS Water Sources . . . . . . . . . . . . . . . 33

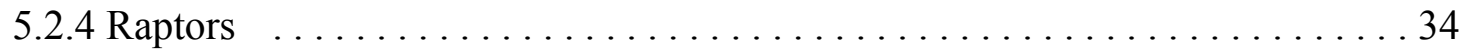

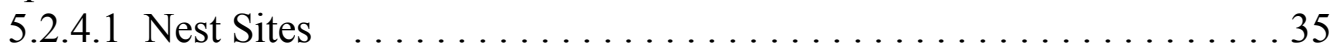

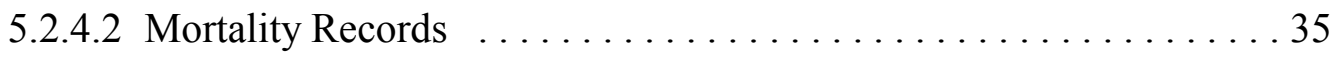




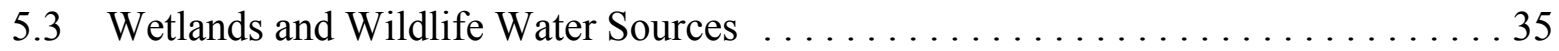

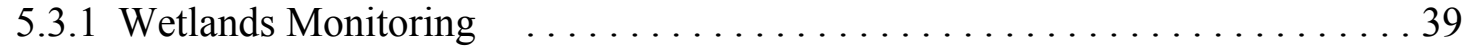

5.3.2 Monitoring of Man-made Water Sources . . . . . . . . . . . . . . . . . . . 39

6.0 MONITORING OF THE HAZMAT SPILL CENTER . . . . . . . . . . . . . . . . . . 45

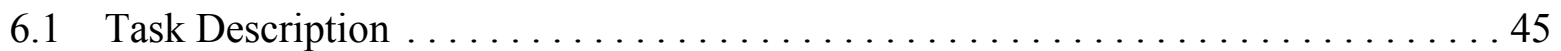

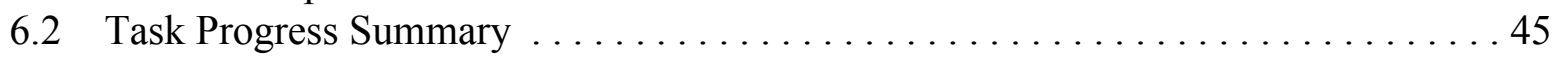

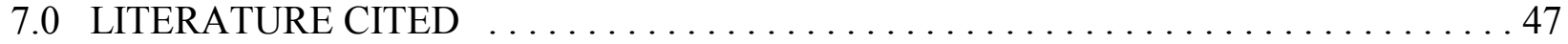

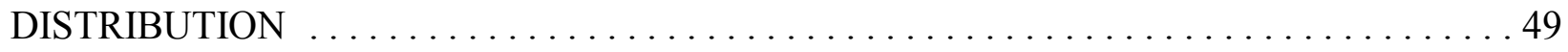




\section{List of Tables}

Table 1. Sensitive species that are protected under state or federal regulations which are

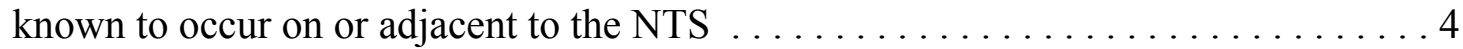

Table 2. Summary of biological surveys conducted on the NTS during FY $2002 \ldots \ldots \ldots$

Table 3. FY 2002 projects within important habitats and acreage proposed

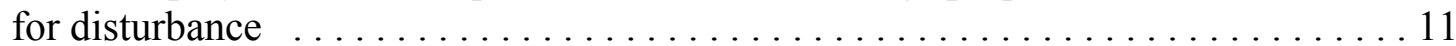

Table 4. Total acreage proposed for disturbance within important habitats over the past four

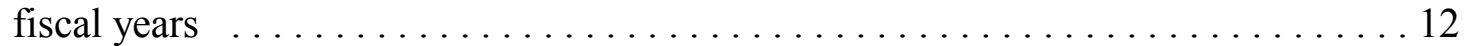

Table 5. Summary of tortoise compliance activities conducted by BN biologists during

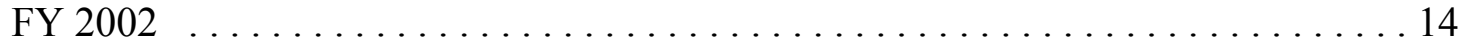

Table 6. Parameters and threshold values for desert tortoise monitoring on the NTS . . . . 16

Table 7. Characteristics of E. concinnum habitat on the NTS . . . . . . . . . . . 24

Table 8. Number of horse individuals observed on the NTS by age class, gender, and

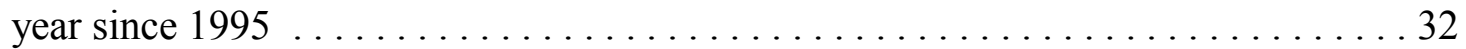

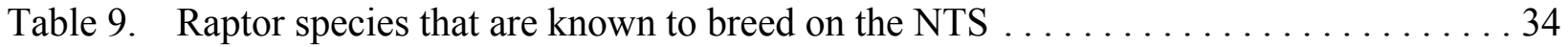

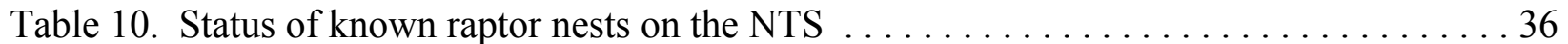

Table 11. Summary of NTS raptor mortality records from $1990-2002 \ldots \ldots \ldots \ldots$

Table 12. Seasonal data from selected natural water sources on the NTS collected during

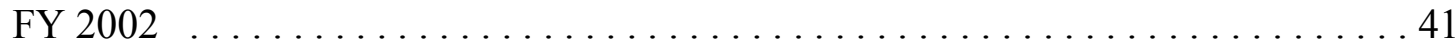

Table 13. Seasonal wildlife use at selected natural water sources on the NTS during FY 2002

\section{List of Figures}

Figure 1. Biological surveys conducted on the NTS during FY $2002 \ldots \ldots \ldots \ldots \ldots \ldots$

Figure 2. Biological surveys conducted in important habitats of the NTS during FY $2002 \ldots 10$

Figure 3. Sensitive plant populations monitored on the NTS during FY 2002 . . . . . . . 22

Figure 4. Density of ten populations of $A$. beatleyae from 1989 to 2002 . . . . . . . . . 23

Figure 5. Known western burrowing owl distribution on the NTS $\ldots \ldots \ldots \ldots \ldots \ldots 27$

Figure $6 . \quad$ Known bat roost sites on the NTS . . . . . . . . . . . . . . . . . . . . . 29

Figure 7. Feral horse sightings and horse sign observed on the NTS during FY $2002 \ldots \ldots 1$

Figure 8. Raptor nests monitored on the NTS during FY $2002 \ldots \ldots \ldots \ldots \ldots \ldots$

Figure 9. Natural water sources on the NTS sampled during FY $2002 \ldots \ldots \ldots \ldots \ldots \ldots$

Figure 10. Man-made water sources monitored for wildlife use and mortality on the NTS

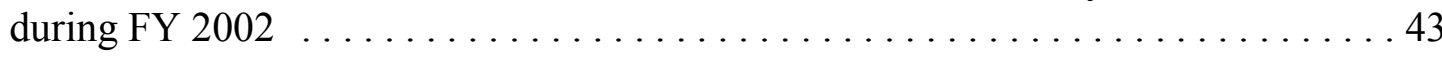


THIS PAGE IS INTENTIONALLY LEFT BLANK 


\section{ACRONYMS AND ABBREVIATIONS}

\begin{tabular}{|c|c|}
\hline $\mathrm{BN}$ & Bechtel Nevada \\
\hline CAU & Corrective Action Unit \\
\hline CAS & Corrective Action Site \\
\hline CWA & Clean Water Act \\
\hline DAF & Device Assembly Facility \\
\hline $\mathrm{DOE} / \mathrm{NV}$ & U.S. Department of Energy, Nevada Operations Office \\
\hline EGIS & Ecological Geographic Information System \\
\hline ELU & Ecological Landform Unit \\
\hline EMAC & Ecological Monitoring and Compliance \\
\hline E-MAD & Engine Maintenance, Assembly, and Disassembly \\
\hline ESA & Endangered Species Act \\
\hline ESHD & Environment, Safety, and Health Division \\
\hline FWS & U.S. Fish and Wildlife Service \\
\hline FY & Fiscal Year \\
\hline GPS & Global Positioning System \\
\hline NNHP & Nevada Natural Heritage Program \\
\hline NNPS & Nevada Native Plant Society \\
\hline \multirow[t]{2}{*}{ NNSA/NV } & U.S. Department of Energy, National Nuclear Security Administration \\
\hline & Nevada Operations Office \\
\hline NTS & Nevada Test Site \\
\hline RMP & Resource Management Plan \\
\hline UGTA & Underground Test Area \\
\hline
\end{tabular}


THIS PAGE IS INTENTIONALLY LEFT BLANK 


\section{ABSTRACT}

The Ecological Monitoring and Compliance program, funded through the U.S. Department of Energy, National Nuclear Security Administration Nevada Operations Office, monitors the ecosystem of the Nevada Test Site (NTS) and ensures compliance with laws and regulations pertaining to NTS biota. This report summarizes the program's activities conducted by Bechtel Nevada (BN) during fiscal year 2002. Program activities included: (1) biological surveys at proposed construction sites, (2) desert tortoise compliance, (3) ecosystem mapping and data management, (4) sensitive species and unique habitat monitoring, and (5) biological monitoring at the HAZMAT Spill Center. Biological surveys for the presence of sensitive species and important biological resources were conducted for 26 NTS projects. These projects have the potential to disturb a total of 374 acres. Thirteen of the projects were in desert tortoise habitat, and 13.38 acres of desert tortoise habitat were disturbed. No tortoises were found in or displaced from project areas, and no tortoises were accidentally injured or killed at project areas or along paved roads. Compilation of historical wildlife data continued this year in efforts to develop faunal distribution maps for the NTS. Photographs associated with the NTS ecological landform units sampled to create the NTS vegetation maps were cataloged for future retrieval and analysis. The list of sensitive plant species for which long-term population monitoring is scheduled was revised. Six vascular plants and five mosses were added to the list. Plant density estimates from ten populations of Astragalus beatleyae were collected, and eight known populations of Eriogonum concinnum were visited to assess plant and habitat status. Minimal field monitoring of western burrowing owl burrows occurred. A report relating to the ecology of the western burrowing owl on the Nevada Test Site was prepared which summarizes four years of data collected on this species' distribution, burrow use, reproduction, activity patterns, and food habits. Bat roost sites within seven buildings slated for demolition were identified, and a BN biologist was a contributing author of the Nevada Bat Conservation Plan published by the Nevada Bat Working Group. Thirty-three adult horses and five foals were counted this year. Six active raptor nests (two American kestrel, two Red-tailed hawk, and two Great-horned owl nests) were found and monitored this year. Selected wetlands and man-made water sources were monitored for physical parameters and wildlife use. No dead animals were observed this year in any plastic-lined sump. The chemical release test plan for one experiment at the HAZMAT Spill Center on Frenchman Lake playa was reviewed. Seasonal sampling of downwind and upwind transects near the spill center was conducted to document baseline conditions of biota. 
THIS PAGE IS INTENTIONALLY LEFT BLANK 


\subsection{INTRODUCTION}

In accordance with DOE Order 450.1 "Environmental Protection Program", the Environment, Safety, and Health Division (ESHD) of the U.S. Department of Energy, National Nuclear Security Administration Nevada Operations Office (NNSA/NV) requires ecological monitoring and biological compliance support for activities and programs conducted at the Nevada Test Site (NTS). Bechtel Nevada (BN) Ecological Services has implemented the Ecological Monitoring and Compliance (EMAC) program to provide this support. EMAC is designed to ensure compliance with applicable laws and regulations, delineate and define NTS ecosystems, and provide ecological information that can be used to predict and evaluate the potential impacts of proposed projects and programs on those ecosystems.

The ecological monitoring tasks conducted in fiscal year (FY) 2002 (October 1, 2001, through September 30, 2002) included: (1) Biological Surveys, (2) Desert Tortoise Compliance, (3) Ecosystem Mapping/Data Management, (4) Sensitive Species and Habitat Monitoring, and (5) HAZMAT Spill Center Monitoring. The five sections of this report document work performed under these five program areas. 
THIS PAGE IS INTENTIONALLY LEFT BLANK 


\subsection{BIOLOGICAL SURVEYS}

Biological surveys are performed at proposed NTS project sites where land disturbance will occur. The goal is to minimize adverse effects of land disturbance on sensitive plant and animal species, their associated habitat, and important biological resources. Sensitive species include those protected under state or federal regulations which are known or suspected to occur on the NTS (Table 1). Important biological resources include such things as cover sites, nest or burrow sites, roost sites, or water sources important to sensitive species. Survey reports are written to document species and resources found and to provide mitigation recommendations.

\subsection{Sites Surveyed and Sensitive Species Observed}

Biological surveys for 26 projects were conducted on or near the NTS (Figure 1, Table 2). For some of the projects, multiple sites were surveyed (Figure 1). A total of 629.96 acres was surveyed for the projects (Table 2).

Thirteen of the projects had sites within the range of the threatened desert tortoise (Gopherus agassizii) (Figure 1). Sensitive species (or their sign) and important biological resources found within proposed project boundaries included an active Great Horned Owl nest, active and inactive predator burrows, and mature yucca and cacti (Table 2). A pair of breeding Great Horned Owls was found in a building scheduled for demolition (Nest A3-B2, see Section 5.2.4). Demolition of this building was delayed until the owl chicks fledged. BN provided a written summary report of all survey findings and mitigation recommendations, where appropriate (Table 2).

\subsection{Potential Habitat Disturbance}

Six of the projects for which surveys were conducted were entirely on sites previously disturbed (e.g., industrial waste sites, building sites, existing borrow areas, existing well pads, road renovations) (Table 2). Surveys are conducted at old industrial or nuclear weapons testing sites whenever vegetation has reinvaded a site or it is suspected that a sensitive species may be found. For example, tortoises may move through revegetated earthen sumps and may be concealed under vegetation during activities where heavy equipment is used. Preactivity surveys are conducted at such revegetated sites to ensure that they are not in harms way. Also, burrowing owls frequently inhabit burrows and culverts at disturbed sites, so preactivity surveys are conducted to ensure that adults, eggs, and nestlings in burrows are not harmed.

Twenty projects were located either partially or entirely in areas that had not been previously disturbed. These projects have the potential to disturb a total of 374.18 acres, where most (231 acres) are within the proposed Munitions Test Range in Dead Horse Flats in Area 18 (Project 02-23) (Table 2). Twelve of the 20 projects that will cause new disturbances occur in areas designated as important habitat on the NTS (Figure 2, Table 3). During vegetation mapping of the NTS, Ecological Landform Units (ELUs) were evaluated and some were identified as pristine, unique, sensitive, and diverse (see definitions, Table 3) (DOE, 1998). A single ELU could be classified as more than one type of important habitat. Figure 2 shows the 
Table 1. Sensitive species that are protected under state or federal regulations which are known to occur on or adjacent to the NTS

\begin{tabular}{|c|c|c|}
\hline Flowering Plant Species & Common Names & Status $^{a}$ \\
\hline Arctomecon merriamii & White bearpoppy & SOC, W, IA \\
\hline Astragalus beatleyae & Beatley's milkvetch & $\mathrm{SOC}, \mathrm{W}, \mathrm{A}$ \\
\hline Astragalus funereus & black woollypod & SOC, W, A \\
\hline Astragalus oopherus var. clokeyanus & Clokey’s egg milkvetch & $\mathrm{SOC}, \mathrm{W}, \mathrm{A}$ \\
\hline Camissonia megalantha & Cane Spring suncup & SOC, W, IA \\
\hline Cymopterus ripleyi var. saniculoides & Ripley's springparsley & SOC, W, IA \\
\hline Eriogonum сопсіпnит & Darin's buckwheat & $\mathrm{W}, \mathrm{A}$ \\
\hline Eriogonum heermannii var. clokeyi & Clokey's buckwheat & $\mathrm{W}, \mathrm{A}$ \\
\hline $\begin{array}{l}\text { Frasera pahutensis or F. albicaulis var. } \\
\text { modocensis }\end{array}$ & $\begin{array}{l}\text { Pahute green gentian or Modoc } \\
\text { elkweed }\end{array}$ & SOC, W, IA \\
\hline Galium hilendiae ssp. kingstonense & Kingston Mountain bedstraw & SOC, W, IA \\
\hline Hulsea vestita ssp. inyoensis & Inyo hulsea & $\mathrm{W}, \mathrm{IA}$ \\
\hline Ivesia arizonica var. saxosa & Whitefeather ivesia & $\mathrm{W}, \mathrm{A}$ \\
\hline Lathyrus hitchcockianus & Hitchcock's peavine & $\mathrm{W}, \mathrm{A}$ \\
\hline Penstemon pahutensis & Pahute penstemon & SOC, W, IA \\
\hline Phacelia beatleyae & Beatley’s phacelia & $\mathrm{SOC}, \mathrm{W}, \mathrm{A}$ \\
\hline Phacelia mustelina & Weasel phacelia & $\mathrm{W}, \mathrm{IA}$ \\
\hline Phacelia parishii & Parish's phacelia & SOC, W, IA \\
\hline \multicolumn{3}{|l|}{ Moss Species } \\
\hline Crossidiium seriatum & seriate crossidium & $\mathrm{W}, \mathrm{E}$ \\
\hline Didymodon nevadensis & Gold Butte moss & $\mathrm{W}, \mathrm{E}$ \\
\hline Entosthodon planoconvexus & planoconvex enthosthodon & $\mathrm{W}, \mathrm{E}$ \\
\hline Grimmia americana & American grimmia & $\mathrm{W}, \mathrm{E}$ \\
\hline Trichostomum sweetii & sweet tricohostomum & $\mathrm{W}, \mathrm{E}$ \\
\hline \multicolumn{3}{|l|}{ Reptile Species } \\
\hline Gopherus agassizii & Desert tortoise & LT, NPT \\
\hline Sauromalus obesus & Chuckwalla & SOC \\
\hline \multicolumn{3}{|l|}{ Bird Species $^{b}$} \\
\hline Athene cunicularia hypugea & Western burrowing owl & SOC, $\mathrm{P}$ \\
\hline Alectoris chukar & Chukar & G \\
\hline Aquila chrysaetos & Golden eagle & $\mathrm{EA}, \mathrm{P}$ \\
\hline
\end{tabular}


Table 1. (Continued)

\begin{tabular}{|c|c|c|}
\hline Bird Species & Common Name & Status $^{a}$ \\
\hline Buteo regalis & Ferruginous hawk & SOC, $\mathrm{P}$ \\
\hline Callipepla gambelii & Gambel's quail & $\mathrm{G}$ \\
\hline Charadrius montanus & Mountain plover & $\mathrm{PT}, \mathrm{P}$ \\
\hline Chlidonias niger & Black tern & SOC \\
\hline Empidonax wrightii & Gray flycatcher & $\mathrm{SOC}$ \\
\hline Falco peregrinus anatum & American peregrine falcon & $<$ LE, P \\
\hline Haliaeetus leucocephalus & Bald eagle & LT-PD, EA, P \\
\hline Ixobrychus exillis hesperis & Western least bittern & $\mathrm{SOC}, \mathrm{P}$ \\
\hline Phainopepla nitens & Phainopepla & $\mathrm{SOC}$ \\
\hline Phasianus colchicus & Ring-necked pheasant & G \\
\hline Plegadis chihi & White-faced ibis & SOC, $\mathrm{P}$ \\
\hline \multicolumn{3}{|l|}{ Mammal Species } \\
\hline Antilocapra americana & Pronghorn antelope & $\mathrm{G}$ \\
\hline Corynorhinus townsendii pallescens & Townsend's big-eared bat & $\mathrm{SOC}$ \\
\hline Equus asinus & Burro & $\mathrm{H} \& \mathrm{~B}$ \\
\hline Equus caballus & Horse & $\mathrm{H} \& \mathrm{~B}$ \\
\hline Euderma maculatum & Spotted bat & SOC, NPT \\
\hline Felis concolor & Mountain lion & G \\
\hline Lynx rufus & Bobcat & $\mathrm{F}$ \\
\hline Myotis ciliolabrum & Small-footed myotis & $\mathrm{SOC}$ \\
\hline Myotis evotis & Long-eared myotis & SOC \\
\hline Myotis thysanodes & Fringed myotis & $\mathrm{SOC}$ \\
\hline Myotis volans & Long-legged myotis & SOC \\
\hline Myotis yumanensis & Yuma myotis & $\mathrm{SOC}$ \\
\hline Ovis canadensis nelsoni & Desert bighorn sheep & $\mathrm{G}$ \\
\hline Odocoileus hemionus & Mule deer & G \\
\hline Sylvilagus audubonii & Audubon's cottontail & G \\
\hline Sylvilagus nuttallii & Nuttall's cottontail & G \\
\hline Urocyon cinereoargenteus & Gray fox & $\mathrm{F}$ \\
\hline Vulpes velox macrotis & Kit fox & $\mathrm{F}$ \\
\hline
\end{tabular}


Table 1. (Continued)

a Status Codes:

Endangered Species Act, U.S. Fish and Wildlife Service

LT - Listed Threatened

PT - Proposed for listing as Threatened

PD - Proposed for delisting

RA - Former Candidate or Proposed species; current information does not support proposal to list because species has proven more abundant or widespread, or to lack identifiable threats; a species of concern

$<$ LE - Former listed endangered species

SOC - Species of concern

U.S. Department of Interior

H\&B - Protected under Wild Free Roaming Horses and Burros Act

EA - Protected under Bald and Golden Eagle Act

State of Nevada

NPT - Protected

G - Regulated as game

F - Regulated as fur-bearer

P - Protected bird

Long-term Plant Monitoring Status for Nevada Test Site (NTS) (see Section 5.1.1 of this report)

A - Active

IA - Inactive

E - Evaluate

W - On Nevada Natural Heritage Program's watch list

${ }^{\mathbf{b}}$ Does not include all bird species that are protected by the Migratory Bird Treaty Act or by the state. Additionally, there are 26 birds which have been observed on the NTS, which are all protected by the state. 


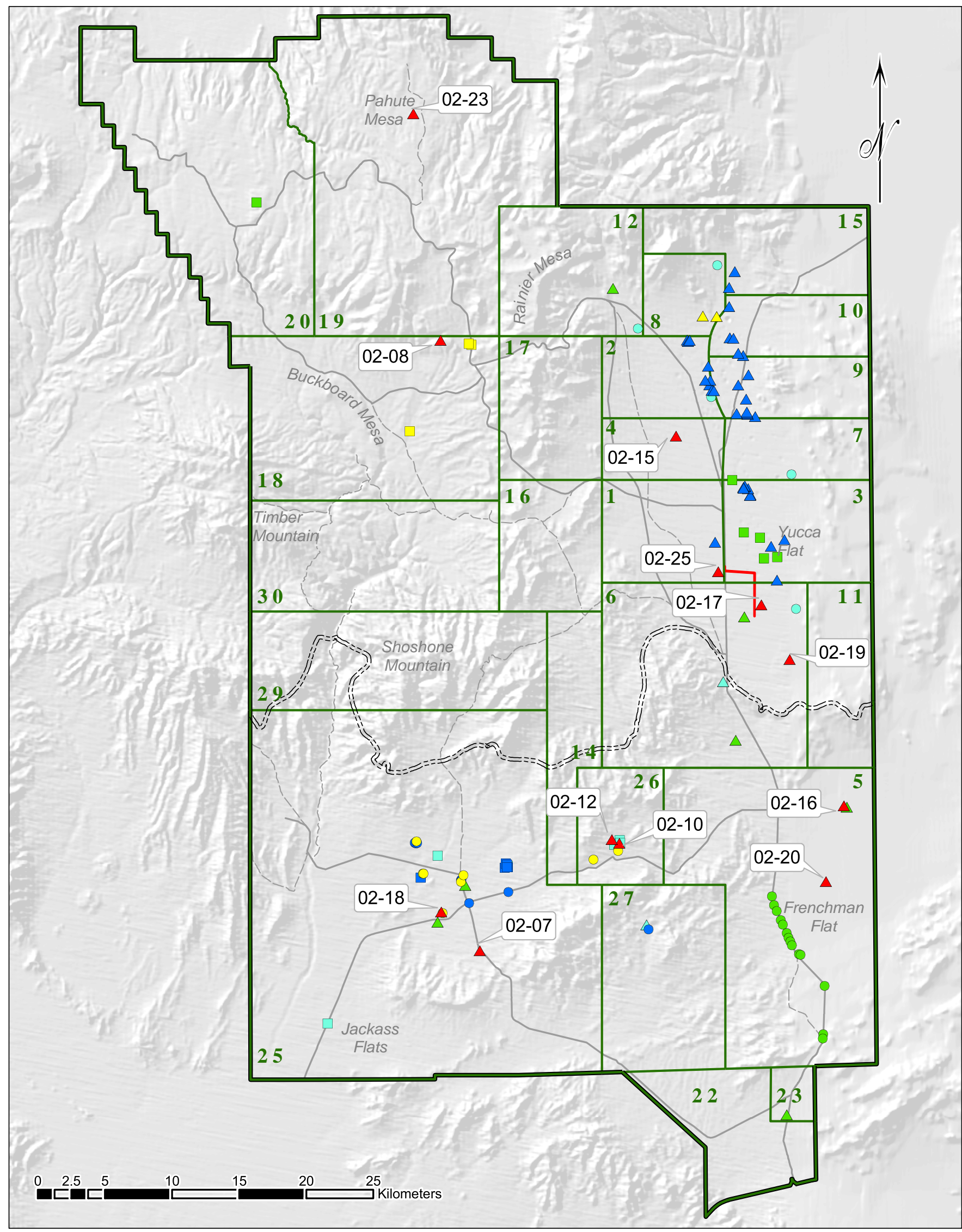

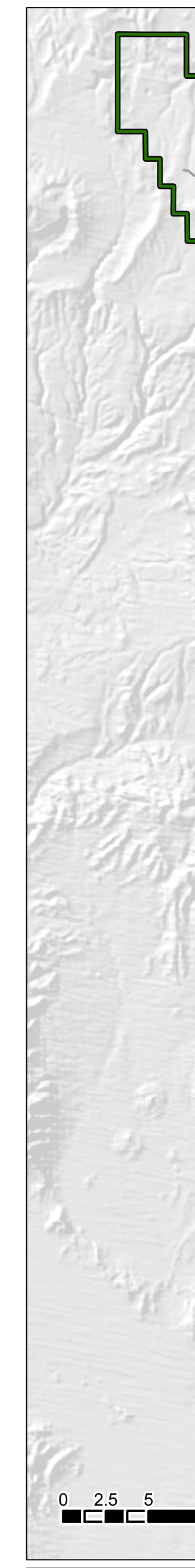

- 02-01 02-04 $\bigcirc \quad 02-09 \bigcirc \quad 02-21 \quad \Delta \quad$ All other surveys $\frown$ Primary roads

$\Delta$ 02-02 $\triangle$ 02-05 $\triangle$ 02-11 $\triangle$ 02-22 02-26 $\backsim$ Secondary roads

- 02-03 $\square \quad 02-06 \square \quad 02-14 \quad \square \quad 02-24$ ご, Desert tortoise northern boundary range

Bechtel Mevada

Figure 1. Biological surveys conducted on the NTS during FY 2002 
Table 2. Summary of biological surveys conducted on the NTS during FY 2002

\begin{tabular}{|c|c|c|c|c|c|}
\hline $\begin{array}{l}\text { Project } \\
\text { No. }\end{array}$ & Project & $\begin{array}{l}\text { Important } \\
\text { Species/ Resources } \\
\text { Found }\end{array}$ & $\begin{array}{l}\text { Area } \\
\text { Surveyed } \\
\text { (acres) }\end{array}$ & $\begin{array}{c}\text { Proposed Project } \\
\text { Area in } \\
\text { Undisturbed } \\
\text { Habitat (acres) }\end{array}$ & $\begin{array}{c}\text { Mitigation } \\
\text { Recommendations }\end{array}$ \\
\hline 02-01 & Corrective Action Unit (CAU) 271 (8 sites) & None & 9.85 & 1.78 & None \\
\hline 02-02 & Borehole Plugging ( 35 sites) & Predator burrow & 32.00 & 0 & Avoid flagged burrow \\
\hline $02-03$ & $\begin{array}{l}\text { Engine Maintenance, Assembly, and Disassembly } \\
\text { (E-MAD) Remediation (CAU 143) (6 sites) }\end{array}$ & Inactive predator burrows & 21.72 & 5.53 & None \\
\hline 02-04 & Mercury Highway Culvert Repairs (14 sites) & Inactive predator burrow & 10.48 & 0.71 & None \\
\hline $02-05$ & New Septic Tanks (7 sites) & Mature yucca, cacti & 29.31 & 4.22 & Avoid yucca, cacti if possible \\
\hline $02-06$ & Mud Pit Disposal Sites (CAU 356) (6 sites) & $\begin{array}{l}\text { Inactive predator burrow, } \\
\text { collapsed kit fox burrow, stick } \\
\text { nest in building }\end{array}$ & 6.13 & 0 & Do not disturb nest \\
\hline 02-07 & Surface Laid Cable & None & 2.08 & 0.07 & None \\
\hline 02-08 & 18-01 Road Renovation & Mature yucca, cacti & 1.48 & 0.56 & Avoid yucca, cacti if possible \\
\hline 02-09 & $\begin{array}{l}\text { Underground Test Area (UGTA) Drill Holes in } \\
\text { Yucca Flat ( } 5 \text { sites) }\end{array}$ & $\begin{array}{l}\text { Mature yucca, cacti, relic } \\
\text { creosote shrub population }\end{array}$ & 59.53 & 39.68 & $\begin{array}{l}\text { Avoid yucca, cacti, and relic creosote } \\
\text { shrubs if possible }\end{array}$ \\
\hline $02-10$ & Phoenix Facility & None & 0.10 & 0.07 & None \\
\hline $02-11$ & Closure of Release Sites (CAU 326) (2 sites) & None & 2.77 & 0.23 & None \\
\hline $02-12$ & Explosive Magazine Move (CANCELLED) & None & 0.20 & 0.10 & None \\
\hline $02-13 *$ & $\begin{array}{l}\text { Fiscal Year } 2002 \text { Building Demolition ( } 64 \\
\text { buildings) }\end{array}$ & $\begin{array}{l}\text { Active Great Horned Owl nest, } \\
\text { inactive Raven nest, } 5 \text { live bats }\end{array}$ & N/A & 0 & $\begin{array}{l}\text { Delay demolition of building until owl } \\
\text { chicks fledge, have biologist remove } \\
\text { roosting bats prior to demolition }\end{array}$ \\
\hline $02-14$ & $\begin{array}{l}\text { Areas } 25 \text { and } 26 \text { Contaminated Materials and } \\
\text { Waste Dumps (CAU 168) ( } 7 \text { sites) }\end{array}$ & $\begin{array}{l}\text { Collapsed tortoise burrow, } \\
\text { mature yucca, cacti }\end{array}$ & 24.47 & 1.07 & Avoid yucca, cacti if possible \\
\hline $02-15$ & WATUSI Project & None & 29.63 & 5.56 & None \\
\hline $02-16$ & $\begin{array}{l}\text { Radioactive Waste Maintenance Site (RWMS) } \\
\text { Expansion }\end{array}$ & None & 1.95 & 1.95 & None \\
\hline $02-17$ & Fill Pipeline, A06 Construction Sump & None & 1.04 & 0 & None \\
\hline
\end{tabular}

*Building locations not shown on Figure 1. 
Table 2. (Continued)

\begin{tabular}{|c|c|c|c|c|c|}
\hline $\begin{array}{l}\text { Project } \\
\text { Number }\end{array}$ & Project & $\begin{array}{c}\text { Important } \\
\text { Species/ Resources } \\
\text { Found }\end{array}$ & $\begin{array}{c}\text { Area } \\
\text { Surveyed } \\
\text { (acres) }\end{array}$ & $\begin{array}{c}\text { Proposed Project } \\
\text { Area in } \\
\text { Undisturbed } \\
\text { Habitat (acres) }\end{array}$ & $\begin{array}{c}\text { Mitigation } \\
\text { Recommendations }\end{array}$ \\
\hline $02-18$ & Area 25 Spill Sites (CAU 398) & None & 0.32 & 0 & None \\
\hline 02-19 & Yucca Lake Runway Repair and Extension & Inactive predator burrows & 14.63 & 13.77 & None \\
\hline $02-20$ & $\begin{array}{l}\text { Hazmat Spill Center Sensors and Communications } \\
\text { System }\end{array}$ & Collapsed burrows & 11.44 & 11.44 & None \\
\hline $02-21$ & CAU 165 (8 sites) & None & 26.59 & 0.07 & None \\
\hline $02-22$ & Radiological Demarcation (2 sites) & Predator burrows, mature cacti & 57.08 & 44.20 & Avoid burrows and cacti if possible \\
\hline $02-23$ & Munitions Test Range & Pronghorn antelope & 241.42 & 231.6 & None \\
\hline $02-24$ & CAU 394 (3 sites) & None & 0.49 & 0.14 & None \\
\hline $02-25$ & U1a 100 Pair Phone Cable Installation & Inactive predator burrows & 1.81 & 1.81 & None \\
\hline $02-26$ & Tweezer Road to U1g Powerline & $\begin{array}{l}\text { Inactive predator burrows, } \\
\text { mule deer, antelope }\end{array}$ & 43.44 & 15.19 & None \\
\hline & Total & & 629.96 & 379.75 & \\
\hline
\end{tabular}




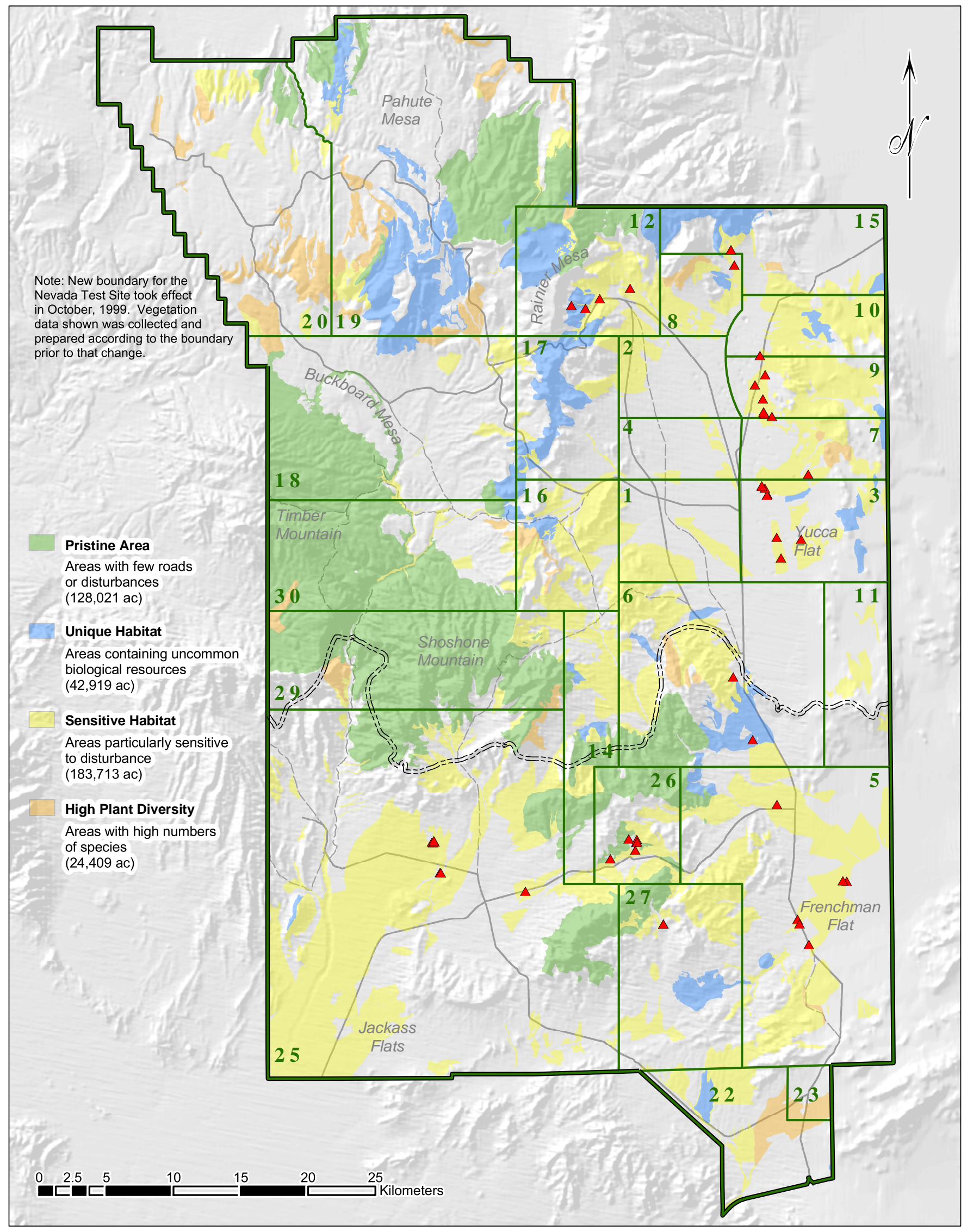

$\begin{aligned} & \text { Biological survey } \\ \text { ミ゙ Desert tortoise northern boundary range } \frown \text { Primary roads } & \smile \text { Secondary roads }\end{aligned}$

Figure $\equiv$ Biological surveys conducted in important habitats of the NTS during FY 2002 
Table 3. FY 2002 projects within important habitats* and acreage proposed for disturbance

\begin{tabular}{|c|c|c|c|c|c|}
\hline $\begin{array}{l}\text { Project } \\
\text { No. }\end{array}$ & Project Name / Site Name (s) & $\begin{array}{c}\text { Pristine } \\
\text { Habitat } \\
\text { (acres) }\end{array}$ & $\begin{array}{c}\text { Unique } \\
\text { Habitat } \\
\text { (acres) }\end{array}$ & $\begin{array}{c}\text { Sensitive } \\
\text { Habitat } \\
\text { (acres) }\end{array}$ & $\begin{array}{l}\text { Diverse } \\
\text { Habitat } \\
\text { (acres) }\end{array}$ \\
\hline 02-01 & $\begin{array}{l}\text { CAU } 271 \text { / Corrective Action Site (CAS) 25-04-08, } \\
\text { CAS 25-04-09 }\end{array}$ & & & 0.15 & 0.15 \\
\hline \multirow[t]{2}{*}{$02-04$} & Mercury Highway Culvert Repairs / Culverts 4, 5 & & & 0.06 & 0.06 \\
\hline & Mercury Highway Culvert Repairs / Culvert 10 & & & & 0.10 \\
\hline \multirow[t]{2}{*}{$02-05$} & New Septic Tanks / Area 6 DAF Septic Tank & & 0.92 & 0.92 & 0.92 \\
\hline & New Septic Tanks / Area 12 Septic Tank & & & 2.77 & 2.77 \\
\hline $02-06$ & $\begin{array}{l}\text { Mud Pit Disposal Sites (CAU 356) / CAS 03-09-03, CAS } \\
03-09-04\end{array}$ & & & 0.25 & 0.25 \\
\hline 02-09 & UGTA Drill Holes in Yucca Flat / Wells ER-7-1, ER-8-1 & & & 24.19 & 24.19 \\
\hline $02-10$ & Phoenix Facility & & & 0.07 & 0.07 \\
\hline $02-12$ & Explosive Magazine Move (Cancelled this FY) & 0.10 & & 0.10 & 0.10 \\
\hline $02-14$ & $\begin{array}{l}\text { Areas } 25 \text { and } 26 \text { Contaminated Materials and Waste } \\
\text { Dumps (CAU 168) / CAS 26-17-10, CAS 26-08-01 }\end{array}$ & & & 0.05 & 0.05 \\
\hline $02-20$ & $\begin{array}{l}\text { Hazmat Spill Center Sensors and Communications System } \\
\text { / Fiberoptic Spur From Building 5-08 }\end{array}$ & & & 11.44 & 11.44 \\
\hline $02-21$ & $\begin{array}{l}\text { CAU } 165 \text { / CAS 25-51-02, CAS 26-07-01, } \\
\text { CAS 26-59-01 }\end{array}$ & 0.05 & & 0.05 & 0.05 \\
\hline \multirow[t]{2}{*}{$02-26$} & Portion of U1g Primary Incoming Power Line & & & 4.04 & 4.04 \\
\hline & Total & 0.15 & 0.92 & 44.09 & 44.19 \\
\hline $\begin{array}{r}\text { Importan } \\
\text { Pris } \\
\text { Uni } \\
\text { Sen } \\
\text { Div }\end{array}$ & $\begin{array}{l}\text { Iabitat Definitions: } \\
\text { e: Habitat with few man-made disturbances } \\
\text { e: Habitat containing uncommon biological resources such } \\
\text { ive: Habitat containing vegetation associations which recove } \\
\text { e: Habitat with high plant species diversity }\end{array}$ & $\begin{array}{l}\text { s a natural } \\
\text { very slowl }\end{array}$ & $\begin{array}{l}\text { om direct } \\
\text { tland }\end{array}$ & turbance & \\
\hline
\end{tabular}


distribution of these important habitats which were ranked so that pristine habitat overlays unique habitat, which then overlays sensitive habitat, which then overlays diverse habitats. The expected acreage to be disturbed in pristine, unique, sensitive, and diverse habitats due to FY 2002 projects is $0.15,0.92,44.09$, and 44.19 respectively (Table 3 ). Note that several projects fall within ELUs having multiple designations (e.g., Project Number 02-05 is located in an ELU classified as diverse, sensitive, and unique). Since FY 1999, when these important habitats were identified during mapping of vegetation associations on the NTS (DOE, 1998), a tally of all acreage proposed for disturbance within them has been kept (Table 4). The tally of acreage that may be disturbed within the four important habitat types defined in Tables 3 and 4 may be used in the future to estimate the area and rate of establishment of invasive species into these habitats. Land-disturbing activities are known to cause the spread of invasive species such as Bromus rubens into areas of the NTS where they have not previously occurred. Such non-native weeds can degrade important habitats by decreasing plant biodiversity and increasing the risk and spread of wildfires. The monitoring and control of invasive plants on federal lands is encouraged under an Executive Order.

Table 4. Total acreage proposed for disturbance within important habitats* over the past four fiscal years. The number of projects within each habitat type per year is shown in parentheses.

\begin{tabular}{ccccc}
\hline Fiscal Year & Pristine Habitat & Unique Habitat & Sensitive Habitat & Diverse Habitat \\
\hline 1999 & 0 & 0 & $78.51(6)$ & $79.97(9)$ \\
2000 & $18.80(2)$ & $10.28(2)$ & $47.84(6)$ & $55.06(8)$ \\
2001 & 0 & $8.65(1)$ & $14.63(3)$ & $14.63(3)$ \\
2002 & $0.15(2)$ & $0.92(1)$ & $44.09(11)$ & $44.19(11)$ \\
& & & & \\
Total & 18.95 & 19.85 & -185.07 & 193.85 \\
\hline \hline
\end{tabular}

*Important Habitat Definitions: see Table 3 


\subsection{DESERT TORTOISE COMPLIANCE}

The desert tortoise occurs within the southern one-third of the NTS. This species is listed as threatened under the Endangered Species Act (ESA). In December 1995, DOE/NV completed consultation with the U.S. Fish and Wildlife Service (FWS) concerning the effects of NNSA/NV activities, described in the Final Environmental Impact Statement for the Nevada Test Site and Off-Site Locations in the State of Nevada (DOE/NV, 1996), on the desert tortoise. A final Biological Opinion (Opinion) (FWS, 1996) was received from the FWS in August 1996. The Opinion concluded that the proposed activities on the NTS were not likely to jeopardize the continued existence of the Mojave population of the species and that no critical habitat would be destroyed or adversely modified. All terms and conditions listed in the Opinion must be followed when activities are conducted within the range of the desert tortoise on the NTS.

The Desert Tortoise Compliance task of EMAC was developed to implement the terms and conditions of the Opinion, to document compliance actions taken by NNSA/NV, and to assist NNSA/NV in FWS consultations. The terms and conditions that were implemented for NNSA/NV by BN staff biologists in FY 2002 included (a) conducting clearance surveys at project sites within 24 hours from the start of project construction, (b) ensuring that environmental monitors are on-site during heavy equipment operation, and (c) preparing an annual compliance report submitted to the FWS.

\subsection{Project-specific Compliance Activities}

In FY 2002, biologists conducted desert tortoise clearance surveys prior to ground-disturbing activities for 13 proposed NTS projects at 57 different sites (Table 5, Figure 1). All but one of the projects (Project Number 02-05) were in, or immediately adjacent to, existing facilities and disturbances. Only one collapsed tortoise burrow was found among all 57 sites surveyed (Table 2, Project Number 02-14). BN Ecological Services ensured that on-site construction monitoring was conducted by a designated environmental monitor at all sites where clearance surveys were performed.

Post-activity surveys to quantify the acreage of tortoise habitat actually disturbed were conducted for four FY 2001 projects and for nine FY 2002 projects (Table 5). Post-activity surveys were not conducted if viable tortoise habitat was not found within the project area boundaries during the clearance survey and if the environmental monitor documented that the project stayed within its proposed boundaries. This fiscal year, a total of 34.26 acres of disturbed tortoise habitat were documented, of which, 13.38 acres were for projects initiated in FY 2002 (Table 5).

\subsection{Other Compliance Activities}

In January, BN submitted to ESHD the annual report that summarized tortoise compliance activities conducted on the NTS from January 1 through December 31, 2001. This report, required under the Opinion, contains (a) the location and size of land disturbances that occurred within the range of the desert tortoise during the reporting period; (b) the number of desert 
Table 5. Summary of tortoise compliance activities conducted by BN biologists during FY 2002

\begin{tabular}{|c|c|c|c|}
\hline $\begin{array}{c}\text { Project } \\
\text { Number }\end{array}$ & Project & Compliance Activities & $\begin{array}{r}\text { Tortoise Habitat } \\
\text { Disturbed (acres) }\end{array}$ \\
\hline $01-09 *$ & $\begin{array}{l}\text { Remediation at Area } 22 \text { Sewage } \\
\text { Lagoons and Desert Rock Airport } \\
\text { Strainer Box (CAU 230/320) }\end{array}$ & Post-activity survey & 0 \\
\hline $01-13^{*}$ & Erosion Control at Area 27 Landfill & Post-activity survey & 0.09 \\
\hline $01-17 *$ & Renovation of Mercury Highway & Post-activity survey & 0.39 \\
\hline $01-21^{*}$ & Frenchman Flat Geo-Seismic Study & Post-activity survey & 20.40 \\
\hline $02-01$ & $\begin{array}{l}\text { CAU } 271 \\
\text { (8 sites) }\end{array}$ & $\begin{array}{l}100 \text { percent-coverage survey, } \\
\text { post-activity survey }\end{array}$ & 1.63 \\
\hline $02-03$ & $\begin{array}{l}\text { E-MAD Remediation (CAU 143) } \\
\text { (6 sites) }\end{array}$ & $\begin{array}{l}100 \text { percent-coverage survey, } \\
\text { post-activity survey }\end{array}$ & 5.53 \\
\hline 02-04 & $\begin{array}{l}\text { Mercury Highway Culvert Repairs } \\
\text { (14 sites) }\end{array}$ & $\begin{array}{l}100 \text { percent-coverage survey, } \\
\text { post-activity survey }\end{array}$ & 0.71 \\
\hline $02-05$ & $\begin{array}{l}\text { New Septic Tanks } \\
\text { (6 sites) }\end{array}$ & $\begin{array}{l}100 \text { percent-coverage survey, } \\
\text { post-activity survey }\end{array}$ & 4.15 \\
\hline $02-07$ & $\begin{array}{l}\text { Surface Laid Cable } \\
\text { (1 site) }\end{array}$ & $\begin{array}{l}100 \text { percent-coverage survey, } \\
\text { post-activity survey }\end{array}$ & 0.07 \\
\hline $02-10$ & $\begin{array}{l}\text { Phoenix Facility } \\
\text { (1 site) }\end{array}$ & $\begin{array}{l}100 \text { percent-coverage survey, } \\
\text { post-activity survey }\end{array}$ & 0.07 \\
\hline $02-11$ & $\begin{array}{l}\text { Closure of Release Sites (CAU 326) } \\
\text { (2 sites) }\end{array}$ & 100 percent-coverage survey & 0 \\
\hline $02-12$ & $\begin{array}{l}\text { Explosive Magazine Move } \\
\text { (1 site) }\end{array}$ & 100 percent-coverage survey & $\begin{array}{c}\mathrm{N} / \mathrm{A}^{1} \\
\text { (Project cancelled) }\end{array}$ \\
\hline $02-14$ & $\begin{array}{l}\text { Areas } 25 \text { and } 26 \text { Contaminated } \\
\text { Materials and Waste Dumps (CAU } \\
\text { 168) ( } 7 \text { sites) }\end{array}$ & $\begin{array}{l}100 \text { percent-coverage survey, } \\
\text { post-activity survey }\end{array}$ & 1.15 \\
\hline $02-16$ & $\begin{array}{l}\text { RWMS Expansion } \\
\text { (1 site) }\end{array}$ & $\begin{array}{l}\text { Voluntary } 100 \text { percent-coverage } \\
\text { survey, site is in area exempt from } \\
\text { terms and conditions of Biological } \\
\text { Opinion }\end{array}$ & N/A \\
\hline $02-18$ & $\begin{array}{l}\text { Area } 25 \text { Spill Sites (CAU 398) } \\
\text { (1 site) }\end{array}$ & 100 percent-coverage survey & 0 \\
\hline $02-20$ & $\begin{array}{l}\text { Hazmat Spill Center Sensors and } \\
\text { Communications System ( } 1 \text { site })\end{array}$ & $\begin{array}{l}\text { Voluntary } 100 \text { percent-coverage } \\
\text { survey, site is in area exempt from } \\
\text { terms and conditions of Biological } \\
\text { Opinion }\end{array}$ & N/A \\
\hline
\end{tabular}


Table 5. (Continued)

\begin{tabular}{|c|c|c|c|}
\hline Project Number & Project & Compliance Activities & $\begin{array}{l}\text { Tortoise Habitat } \\
\text { Disturbed (acres) }\end{array}$ \\
\hline \multirow[t]{3}{*}{$02-21$} & CAU 165 (8 sites) & $\begin{array}{l}100 \text { percent-coverage } \\
\text { survey, post-activity } \\
\text { survey }\end{array}$ & 0.07 \\
\hline & & Total & 34.26 \\
\hline & & $\begin{array}{l}\text { Total - FY } 2002 \text { Projects } \\
\text { Only }\end{array}$ & 13.38 \\
\hline
\end{tabular}

*Projects reported in FY 2001 for which the acres disturbed were not reported ${ }^{1}$ N/A - Not applicable 
tortoises injured, killed, or removed from project sites; (c) a map showing the location of all tortoises sighted on or near roads on the NTS; and (d) a summary of construction mitigation and monitoring efforts.

Compliance with the Opinion will ensure that the two goals of the NNSA/NV Resource Management Plan are being met; namely, that the desert tortoise is protected on the NTS and that the cumulative impacts on this species are minimized (DOE/NV, 1998). In the Opinion, the FWS has determined that the "incidental take"1 of tortoises on the NTS and the cumulative acreage of tortoise habitat disturbed on the NTS are parameters to be measured and monitored annually. During this FY, the threshold levels established by the FWS for these parameters were not exceeded (Table 6). No desert tortoises were accidentally injured or killed, nor were any captured or displaced from NTS project sites.

Table 6. Parameters and threshold values for desert tortoise monitoring on the NTS

\begin{tabular}{|c|c|c|c|}
\hline Monitored Parameter & $\begin{array}{l}\text { Threshold } \\
\text { Value }\end{array}$ & $\begin{array}{c}\text { Adaptive Management } \\
\text { Action }\end{array}$ & $\begin{array}{l}\text { FY } 2002 \text { Value } \\
\text { of Monitored } \\
\text { Parameter }\end{array}$ \\
\hline $\begin{array}{l}\text { Number of tortoises accidentally injured or killed as a } \\
\text { result of NTS activities per year }\end{array}$ & 3 & $\begin{array}{l}\text { Reinitiate consultation with } \\
\text { FWS }\end{array}$ & 0 \\
\hline $\begin{array}{l}\text { Number of tortoises captured and displaced from NTS } \\
\text { project sites per year }\end{array}$ & 10 & $\begin{array}{l}\text { Reinitiate consultation with } \\
\text { FWS }\end{array}$ & 0 \\
\hline $\begin{array}{l}\text { Number of tortoises taken in form of injury or } \\
\text { mortality on paved roads on the NTS by vehicles other } \\
\text { than those in use during a project }\end{array}$ & Unlimited & $\begin{array}{l}\text { Supplemental employee } \\
\text { education and bulletins }\end{array}$ & 1 \\
\hline $\begin{array}{l}\text { Number of total acres of desert tortoise habitat } \\
\text { disturbed during NTS project construction since } 1992\end{array}$ & 3,015 & $\begin{array}{l}\text { Reinitiate consultation with } \\
\text { FWS }\end{array}$ & 212 \\
\hline
\end{tabular}

${ }^{1}$ To "take" a threatened or endangered species, as defined by the ESA, is to harass, harm, pursue, hunt, shoot, wound, kill, trap, capture or collect, or attempt to engage in any such conduct. 


\subsection{ECOSYSTEM MAPPING/DATA MANAGEMENT}

In FY 1996, efforts were begun to map wildlife and plant habitats of the NTS. Field data were collected, analyzed, and preliminary maps created to show basic habitat features. Databases were developed and linked to geographic information system habitat-physical feature maps. The topical report Classification of Vegetation on the Nevada Test Site (Ostler et al., 2000) was published and distributed in FY 2001. Ten vegetation alliances and 20 associations were recognized as occurring on the NTS.

Emphasis during FY 2002 was on the identification and collection of published wildlife data to provide information about wildlife that can be correlated with vegetation alliances and associations. Information about historical species-specific wildlife collection and sighting data from the NTS has been entered into supporting databases that can be linked to the vegetation and site data. Additionally, metadata was prepared to help document the status of site field data and photographs, to identify future gaps of information, and to direct future field work for areas not surveyed previously.

\subsection{Compilation of Historical Wildlife Data}

This year, work started on entering location coordinates into the Ecological Geographic Information System (EGIS) fauna database for historical animal sighting and specimen collection sites on the NTS. The data will be used to link animal distribution data to the vegetation classification data gathered from ELUs. A review of all published vertebrate and invertebrate inventories and research performed on the NTS was conducted to identify geographical information. Other sources searched included field notes from past and present researchers on the NTS and collection records for vertebrate specimens maintained at the Brigham Young University museum in Provo, Utah. Wildlife observations made by BN biologists or reported to Ecological Services by NTS workers are also maintained in the EGIS animal database, and new wildlife observations were entered into the EGIS database as well. To date, thousands of data entries have been made. This work will continue next fiscal year and faunal distribution maps will begin to be produced.

\subsection{NTS Vegetation Classification Metadata}

Metadata associated with the topical report Classification of Vegetation on the Nevada Test Site (Ostler et.al., 2000) were prepared to help document the extent of field information collected for ELUs on the NTS. The location and extent of field photographs were reviewed for completeness and, where necessary, digitally scanned from old films and prints. Because of poor lighting conditions during field data collection, double exposures on some rolls of film, and infrequent camera failure, several photographs of ecological landform units were never taken and are currently lacking. It is anticipated that ELUs without photo documentation will be visited in the future to obtain photographs in order to provide a complete photo coverage of selected ELUs on the NTS. These photographs document site conditions and provide information needed to evaluate habitat for wildlife use. They also provide details of plant community structure, such as shrub height, foliar density, and vertical stratification of the site's vegetation. 
Digital image files of individual ELUs were renamed and centralized into one subdirectory from several dozen compact disks to facilitate the future retrieval of site photos by ELU number and for electronic linkage with EGIS.

Rectified images of 1:24000 scale aerial digital images of the NTS were secured to provide basemaps for correction of vegetation unit polygons and registration with the georectified base-map images. It is anticipated that location of sampling transects as previously gathered by the old Global Positioning System (GPS) hardware (accurate to within 100 meters) will be corrected to more accurately reflect their proper location. This will also enable the more accurate location of sensitive plant populations.

\subsection{Coordination With Ecosystem Management Agencies/Scientists}

Collaboration with the U.S. Geological Survey Biological Services continued in FY 2002. Data that were being gathered will be used to evaluate changes in vegetation originally sampled by Janice Beatley in the 1970s. Data show that significant changes to species and plant community composition have occurred in some areas. Studies will be useful to document changes due to climatic shifts (e.g., global warming) and direct and indirect effects of nuclear testing. New findings will provide needed information to calculate fire risks to NTS vegetation (e.g., the conversion of blackbrush and mixed shrublands to annual grasslands).

Data collected as part of the vegetation mapping efforts was used in support of studies to characterize potential biointrusion into buried waste at the NTS from ants and termites. BN scientists spent several days assisting scientists from Neptune and Company, Inc., of Los Alamos, New Mexico, and scientists at the University of Toronto in Ontario, Canada, in conducting their research efforts. Additional habitat locations were identified in areas dominated by big sagebrush (Artemisia tridentata) with deep soils. Observations of plant root distribution and biomass were also made during the digging of new disposal pit at the Area 5 Radioactive Waste Management Site. Information about vegetation biomass will be summarized in a report during FY 2003. 


\subsection{SENSITIVE SPECIES AND HABITAT MONITORING}

There are 22 plants and 34 animals which occur on the NTS that are considered sensitive because they are either: (a) listed as threatened or endangered under the ESA, (b) current candidates for listing, (c) species of concern to FWS or state agencies, (d) or state-managed species (Table 1). The desert tortoise is the only threatened or endangered species which could be significantly impacted by NNSA/NV activities. EMAC tasks related to the desert tortoise are addressed in Section 3.0 of this report. As with the desert tortoise, the goal of species and habitat monitoring is to ensure the continued presence of all sensitive plants and animals on the NTS by protecting them from significant impacts due to NNSA/NV actions. A secondary goal is to gather sufficient information on these species' distribution and abundance on the NTS to determine if further protection/management under state or federal law is necessary. Sensitive species monitoring tasks include field surveys to identify species' distribution and abundance and monitoring of the known population locations, roost sites, and burrows of these species.

\subsection{Sensitive Plants Species}

In 1998, DOE/NV prepared a Resource Management Plan (RMP) with the objective to protect and conserve sensitive plant species found on the NTS and to minimize cumulative impacts to those species as a result of U.S. Department of Energy (DOE) activities (DOE/NV, 1998). Pursuant to that document, BN published and distributed an Adaptive Management Plan for Sensitive Plant Species on the Nevada Test Site (BN, 2001a). This document presents the procedures designed to ensure that the RMP goals are met by identifying parameters to be measured during long-term monitoring and outlining management actions that may be taken if significant threats to sensitive species are detected.

\subsubsection{Revised List of Sensitive Plant Species for the NTS}

One of the first tasks identified in the adaptive management plan is to identify those plant species found on the NTS that may require protection because of such factors as rarity, susceptibility to disturbance, or importance. Plants known to occur on the NTS and listed by the FWS as endangered, threatened or as a species of concern, are included on the list of sensitive plant species for the NTS. Other agencies are also consulted in determining which species should be protected. The Department of Conservation and Natural Resources of the Nevada Natural Heritage Program (NNHP) maintains a detailed list of rare plants and lichens. The list includes plants protected by all federal agencies, the Division of Forestry of the state of Nevada, and the Nevada Native Plant society. Any species included in their list and known or suspected to occur on the NTS are considered as sensitive plant species for the NTS.

The list of sensitive plant species being monitored on the NTS (BN, 2001b) was reviewed and revised in FY 2002. The revised list is shown in Table 1 (Section 1.0). Two species were removed from the list of sensitive plant species: Penstemon albomarginatus (whitemargin penstemon) and Penstemon fruticiformis var. amargosae (Amargosa penstemon). Both species were originally included on the list because they are known to occur on lands adjacent to the NTS. However, during surveys over the last several years, neither of these species has been 
found. Habitat for these species is not known to occur on the NTS, therefore it is unlikely they will be found in the future.

Six vascular plants and five nonvascular plants were added to the list. All vascular species are known to occur on the NTS and are listed as 'watch' species by the NNHP. None carry federal status. Five species of mosses were added to the NNHP watch list of sensitive species this last year. All are known from collections in southern Nevada and one has been collected on the NTS.

\subsubsection{Long-term Monitoring}

Long-term monitoring of sensitive plant species is part of the adaptive management plan. The goal of this program is to acquire an accurate delineation of populations of sensitive plant species on the NTS and to periodically assess their status for conservation and management purposes. All sensitive plant species (Table 1) were categorized as a species (a) to be monitored, (b) not to be monitored, or (c) to be evaluated. Species that will be monitored are classified as "active" (A) in Table 1 and include those known to occur on the NTS, are on the FWS or NNHP list of sensitive plant species, and have limited distribution either on the NTS or its entire range. Those species in Table 1 classified as "inactive" (IA) will not be monitored under the long-term monitoring plan for NTS plant species (although their presence at proposed project sites during biological surveys are still documented). They include species that are known to occur on the NTS, are has been gathered to suggest that they have widespread distribution on the NTS, in Nevada, or over the western United States. Species classified as "evaluate" (E) in Table 1 include those for which there is insufficient information to determine if they occur on the NTS and whether their distribution or abundance warrants their protection and monitoring. The revised list of sensitive plant species on the NTS includes ten species that will be monitored, five which will be evaluated, and seven which will not be monitored (Table 1). Six of the ten species that will be monitored are annual forbs, three are perennial forbs, and one is a perennial shrub. All five of the species to be evaluated are bryophytes (mosses).

Field monitoring to assess population status is to be conducted for each "active" species at least once every five years. A minimum of two species are selected each year and a representative number of populations are monitored. Population locations and habitat have been described during previous field studies (Blomquist et al. 1992, Blomquist et al. 1995) for many species, so the amount of field description data gathered during long-term monitoring will vary by species based on need. Other data will be collected during field monitoring to ascertain the current status of the species and may include density of plants, evidence of herbivory, disease, or disturbance.

Growing conditions this fiscal year were poor. Sporadic and light winter and spring rains did not provide adequate moisture for germination of annuals or growth of perennials. Only two species were selected to be monitored this year: Astragalus beatleyae (Beatley's milkvetch), a perennial forb, and Eriogonum concinnum (Darwin's buckwheat), an annual forb. No sensitive plant evaluations were scheduled for this fiscal year. Several mosses were collected during field surveys for A. beatleyae and E. concinnum but identifications have not been made. 


\subsubsection{Astragalus beatleyae}

A. beatleyae flowers and sets seed in May and June. Ten of 18 known populations of A. beatleyae were monitored in mid to late June (Figure 3). Characterization of known populations was completed during previous studies. The goal this fiscal year was to collect density estimates of $A$. beatleyae and to note any conditions that may be impacting the plants (e.g., herbivory, disease, etc.). Although growing conditions were not good this year, individuals of $A$. beatleyae were observed during preliminary field surveys.

The number of individuals of $A$. beatleyae within each population was estimated by sampling two permanent transects previously established at each site (Blomquist et al., 1992). Transects were selected based on which transects had the highest number of individuals recorded in 1991 when sampling last occurred. Plant density was higher in 2002 than it was in 1989 at two of the populations ( $2 \mathrm{~b}$ and 8 ) (Figure 4). However, at all other populations, A. beatleyae density was lowest in 2002. This was not unexpected given the poor growing conditions this year and considering there was no evidence of growth of other perennial forbs this year. Documentation of plant densities during these poor growing conditions will serve as a reference for future monitoring. There was no evidence of any of the populations being impacted by DOE activities.

\subsubsection{Eriogonum concinnum}

E. concinnum flowers and sets seed in late summer. Eight known E. concinnum populations were identified from herbarium records and from historic plant location maps of the NTS (Rhoads et al. 1977) (Figure 3). Characterization of E. concinnum populations had not been done. Only brief habitat descriptions are available from NTS herbarium collections made in the 1960s and 1970s. Preliminary surveys of five of the known sites were conducted in early August to determine if there were any living plants this year. Only old stalks from previous years were found. It was not possible to determine if the old stalks were E. concinnum or closely related species of Eriogonum. Based on these preliminary findings, an assessment of the current status of the species was not possible, therefore efforts were focused on relocating the eight historic locations and gathering information to characterize the habitat of E. concinnum.

All eight locations of E. concinnum (Figure 3) were visited in August of 2002. Habitat data were collected at each of the sites (Table 7) and added to the sensitive plant database maintained under the EMAC program. The habitat for E. concinnum is characterized by sandy soils associated with white volcanic tuff. Slopes vary from $>35$ percent to sandy flat bottoms and borrow areas along roads. The woodland and shrubland associations (Ostler et al., 2000) in which the populations are located are presented in Table 7.

Plotting population boundaries of E. concinnum in the field was not done this year due to the poor growing conditions and almost complete absence of E. concinnum. This phase of long-term monitoring will be completed in future years under more favorable growing conditions. Monitoring of population status will continue at a future time when conditions are more favorable for germination and growth. From the preliminary observations this year, it appears that only a fraction of the potential habitat for E. concinnum has been identified on the NTS. Future studies may show this species to be much more widespread than is currently indicated from herbarium records. 


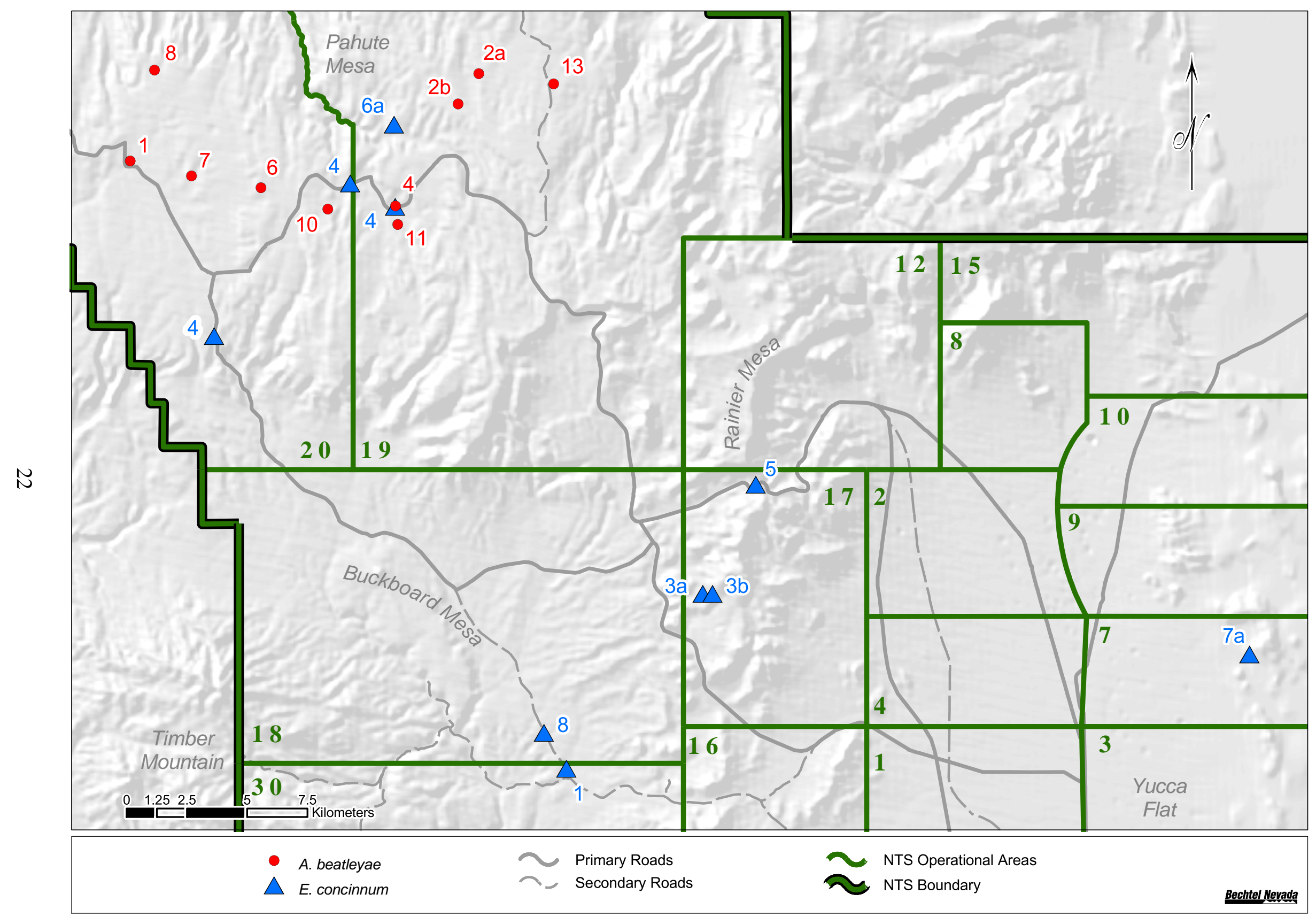

Figure 3. Sensitive plant populations monitored on the NTS during FY 2002 
Figure 4. Density of ten populations of $A$. beatleyae from 1989 to 2002. Site names are same as those used in Blomquist et al. 1992.

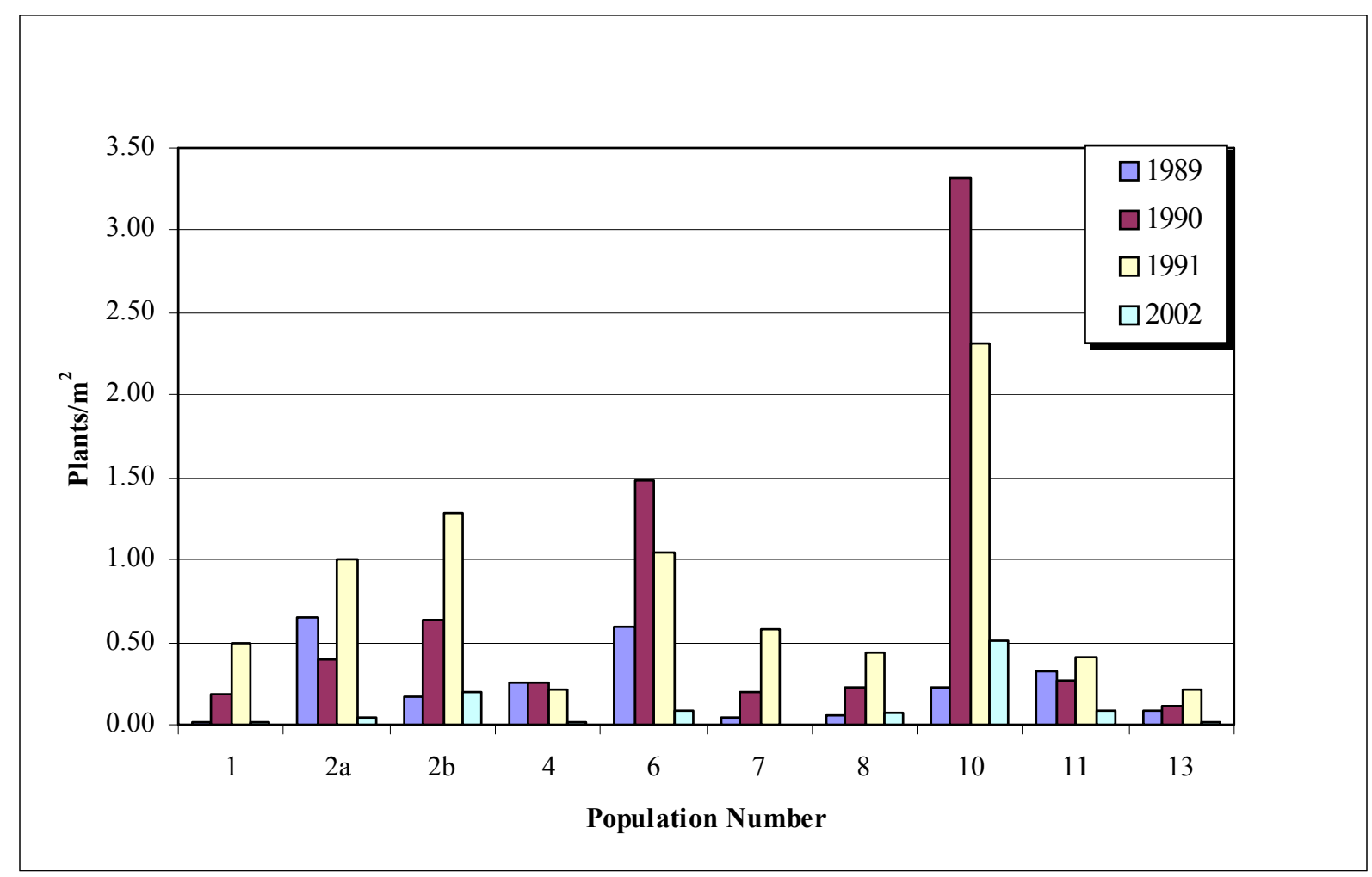


Table 7. Characteristics of $E$. concinnum habitat on the NTS

\begin{tabular}{|c|c|c|c|c|c|c|c|c|}
\hline \multirow[b]{2}{*}{$\begin{array}{l}\text { Habitat } \\
\text { Feature }\end{array}$} & \multicolumn{8}{|c|}{ Plant Population Name and Number } \\
\hline & $\begin{array}{c}\text { Buckboard Mesa } \\
\text { (No. 1) }\end{array}$ & $\begin{array}{l}\text { W Sugar } \\
\text { Loaves } \\
\text { (No. 3a) }\end{array}$ & $\begin{array}{c}\text { WSW } \\
\text { Pinyon } \\
\text { Butte } \\
\text { (No. 3b) }\end{array}$ & $\begin{array}{l}\text { Pahute Mesa } \\
\text { Roadcuts }{ }^{1} \\
\text { (No. 4) }\end{array}$ & $\begin{array}{c}\text { Stockade Wash/ } \\
\text { Holmes Road } \\
\text { Jnct } \\
\text { (No. 5) }\end{array}$ & $\begin{array}{l}\text { E Silent } \\
\text { Canyon } \\
\text { (No. 6a) }\end{array}$ & $\begin{array}{c}\text { NE } \\
\text { Reitmann } \\
\text { Seep } \\
\text { (No. 7a) }\end{array}$ & $\begin{array}{c}\text { Buckboard } \\
\text { Mesa - } \\
\text { Disturbed - } \\
\text { (No. 8) }\end{array}$ \\
\hline Elevation (ft) & 4,850 & 5,800 & 5,965 & 6,160 & 6,400 & 6,620 & 4,660 & 4,925 \\
\hline $\begin{array}{l}\text { Vegetation } \\
\text { Association }^{2}\end{array}$ & $\begin{array}{l}\text { Green } \\
\text { Rabbitbrush- } \\
\text { Nevada Jointfir }\end{array}$ & $\begin{array}{l}\text { Basin Boig } \\
\text { Sagebrush- } \\
\text { Green } \\
\text { Rabbitbrush }\end{array}$ & $\begin{array}{l}\text { Singleleaf } \\
\text { Pinyon- } \\
\text { Black } \\
\text { Sagebrush }\end{array}$ & $\begin{array}{l}\text { Singleleaf Pinyon- } \\
\text { Basin Big Sagebrush, } \\
\text { Basin Big } \\
\text { Sagebrush-Green } \\
\text { Rabbitbrush, Nevada } \\
\text { Jointfir-Spiny } \\
\text { Hopsage }\end{array}$ & Miscellaneous & $\begin{array}{l}\text { Singleleaf } \\
\text { Pinyon- } \\
\text { Black } \\
\text { Sagebrush }\end{array}$ & $\begin{array}{l}\text { Blackbrush } \\
\text {-Nevada } \\
\text { Jointfir }\end{array}$ & $\begin{array}{l}\text { Green } \\
\text { Rabbitbrush- } \\
\text { Nevada } \\
\text { Jointfir }\end{array}$ \\
\hline Soils $^{3}$ & $\begin{array}{l}\text { Loose sand at } \\
\text { base of tan } \\
\text { volcanic cliffs }\end{array}$ & $\begin{array}{l}\text { Volcanic } \\
\text { tuff }\end{array}$ & $\begin{array}{l}\text { Volcanic } \\
\text { tuff hills } \\
\text { and cliffs }\end{array}$ & $\begin{array}{l}\text { Reddish brown / } \\
\text { white rock slopes }\end{array}$ & $\begin{array}{l}\text { Disturbed - soil } \\
\text { derived from light } \\
\text { colored tuff }\end{array}$ & $\begin{array}{l}\text { Volcanic } \\
\text { tuff/ sands }\end{array}$ & $\begin{array}{l}\text { Light } \\
\text { colored } \\
\text { volcanics }\end{array}$ & Disturbed \\
\hline Aspect $^{4}$ & Northeast & West & South & Various & Southwest & South & West & West \\
\hline Slope (\%) ${ }^{4}$ & $10-35$ & $1-10$ & $0-10$ & $35+$ & $0-10$ & $35+$ & $35+$ & $0-10$ \\
\hline $\begin{array}{l}\text { Topographic } \\
\text { Position } 4\end{array}$ & $\begin{array}{l}\text { Mid slope to } \\
\text { bottom }\end{array}$ & Bottom & Bottom & $\begin{array}{l}\text { Lower slope to } \\
\text { bottom }\end{array}$ & Bottom & $\begin{array}{l}\text { Crest to } \\
\text { upper } \\
\text { slope }\end{array}$ & $\begin{array}{l}\text { Mid slope } \\
\text { to upper } \\
\text { slope }\end{array}$ & Bottom \\
\hline Light ${ }^{4}$ & Open & Open & Open & Open & Open & Open & Open & Open \\
\hline $\begin{array}{l}\text { Plant } \\
\text { Abundance }^{3}\end{array}$ & $\begin{array}{l}\text { Common (1968), } \\
\text { Abundant (1969), } \\
\text { Rare (1977) }\end{array}$ & $\begin{array}{l}\text { Abundant } \\
(1969), \\
\text { Scattered } \\
(1978)\end{array}$ & $\begin{array}{l}\text { Common } \\
(1968)\end{array}$ & $\begin{array}{l}\text { Scattered to common } \\
\text { (1977) }\end{array}$ & $\begin{array}{l}\text { Small population } \\
(1969)\end{array}$ & $\begin{array}{l}\text { Common } \\
\text { (1968), } \\
\text { Abundant } \\
\text { (1977) }\end{array}$ & $\begin{array}{l}\text { Common } \\
(1983)\end{array}$ & $\begin{array}{l}\text { Abundant } \\
\text { (1969) }\end{array}$ \\
\hline
\end{tabular}

${ }^{1}$ Three historic locations along a six mile segment of Pahute Mesa Road.

${ }^{2}$ Classified as per Ostler et al., 2000.

${ }^{3}$ Taken from herbarium notes.

${ }^{4}$ Collected during site visits in FY 2002. 